Pacific Journal of Mathematics

THE STRUCTURE OF PURE COMPLETELY BOUNDED AND
COMPLETELY POSITIVE MULTILINEAR OPERATORS 


\title{
THE STRUCTURE OF PURE COMPLETELY BOUNDED AND COMPLETELY POSITIVE MULTILINEAR OPERATORS
}

\section{ZHONG-JIN RUAN}

\begin{abstract}
Let $A$ be a $C^{*}$-algebra and $B(H)$ the algebra of all bounded linear operators on a Hilbert space $H$. We study the structure of pure completely bounded and completely positive multilinear operators from $A^{k}=A \times \cdots \times A$ into $B(H)$.
\end{abstract}

1. Introduction. The definition of completely bounded (resp. completely positive) multilinear operators from one $C^{*}$-algebra into another was introduced by Christensen and Sinclair [4]. We begin by recalling these definitions for our convenience.

Throughout this paper, we assume that $C^{*}$-algebras are unital. Let $A$ and $B$ be $C^{*}$-algebras. We denote $M_{n}(A)=\left\{\left[a_{i j}\right]: a_{i j} \in A\right\}$ (resp. $\left.M_{n}(B)\right)$ the $C^{*}$-algebra of $n \times n$ matrices over $A$ (resp. $B$ ). If $\phi: A^{k}=A \times \cdots \times A \rightarrow B$ is a $k$-linear operator, the $k$-linear operator $\phi_{n}: M_{n}(A)^{k} \rightarrow M_{n}(B)$ is defined by

$$
\phi_{n}\left(A_{1}, A_{2}, \ldots, A_{k}\right)=\left[\sum_{i_{2}, \ldots, i_{k}=1}^{n} \phi\left(a_{i_{1} i_{2}}, a_{i_{2} i_{3}}, \ldots, a_{i_{k} i_{k+1}}\right)\right]
$$

for all $A_{j}=\left[a_{i, i_{j+1}}\right] \in M_{n}(A) \quad(1 \leq j \leq k)$. We define the norm of $\phi_{n}$ by

$$
\begin{aligned}
\left\|\phi_{n}\right\|=\sup \left\{\left\|\phi_{n}\left(A_{1}, A_{2}, \ldots, A_{k}\right)\right\|:\right. & A_{j} \in M_{n}(A) \\
& \text { with } \left.\left\|A_{j}\right\| \leq 1 \text { for } 1 \leq j \leq k\right\}
\end{aligned}
$$

and define the completely bounded norm of $\phi$ by

$$
\|\phi\|_{c b}=\sup \left\{\left\|\phi_{n}\right\|: n \in N\right\} \text {. }
$$

A $k$-linear operator $\phi$ is called completely bounded (resp. completely contractive) if the completely bounded norm $\|\phi\|_{c b}$ is finite (resp. $\left.\|\phi\|_{c b} \leq 1\right)$. We denote $C B\left(A^{k}, B\right)$ the complex Banach space of all completely bounded $k$-linear operators from $A^{k}$ into $B$. If $\phi: A^{k} \rightarrow B$ is a $k$-linear operator, the adjoint $k$-linear operator $\phi^{*}$ from $A^{k}$ into $B$ is defined by

$$
\phi^{*}\left(a_{1}, \ldots, a_{k}\right)=\phi\left(a_{k}^{*}, \ldots, a_{1}^{*}\right)^{*}
$$


for all $a_{1}, \ldots, a_{k} \in A$. If $\phi \in C B\left(A^{k}, B\right)$, then so is $\phi^{*}$ with $\left\|\phi^{*}\right\|_{c b}=\|\phi\|_{c b}$. This gives an involution on the complex Banach space $C B\left(A^{k}, B\right)$. A completely bounded $k$-linear operator $\phi \in C B\left(A^{k}, B\right)$ is called symmetric if $\phi=\phi^{*}$. We denote $C B_{s}\left(A^{k}, B\right)$ the set of all completely bounded symmetric $k$-linear operators from $A^{k}$ into $B$. Then $C B_{S}\left(A^{k}, B\right)$ is just the real space of all selfadjoint elements in $C B\left(A^{k}, B\right)$. $A$ k-linear operator $\phi: A^{k} \rightarrow B$ is called completely positive if:

(i) $k=2 l-1$ odd. We have $\phi_{n}\left(A_{1}^{*}, \ldots, A_{l-1}^{*}, A_{l}, A_{l-1}, \ldots, A_{1}\right)$ $\geq 0$ for all $A_{1}, \ldots, A_{l-1} \in M_{n}(A), A_{l} \in M_{n}(A)^{+}$and all $n \in N$, or

(ii) $k=2 l$ even. We have $\phi_{n}\left(A_{1}^{*}, \ldots, A_{l}^{*}, A_{l}, \ldots, A_{1}\right) \geq 0$ for all $A_{1}, \ldots, A_{l} \in M_{n}(A)$ and all $n \in N$.

We denote $C B^{+}\left(A^{k}, B\right)$ the set of all completely bounded and completely positive $k$-linear operators from $A^{k}$ into $B$. Then $C B^{+}\left(A^{k}, B\right)$ is a proper positive cone in the real Banach space $C B_{s}\left(A^{k}, B\right)$. It is known from [18], [10], [12] and [4] that $C B_{s}\left(A^{k}, B\right)$ $=C B^{+}\left(A^{k}, B\right)-C B^{+}\left(A^{k}, B\right)$ if $B$ is an injective $C^{*}$-algebra. This gives a natural partial ordering on $C B_{S}\left(A^{k}, B\right)$ defined by $\psi \leq \phi$ if $\phi-\psi \in C B^{+}\left(A^{k}, B\right)$.

REMARK. The above definition of completely bounded (resp. completely bounded symmetric, completely positive) $k$-linear operators from $A^{k}$ into $B$ is a natural generalization of the usual definition of completely bounded (resp. completely bounded self-adjoint, completely positive) linear operators from $C^{*}$-algebra $A$ into $C^{*}$-algebra $B$. In the case of $k=1$, we know that every completely positive linear operator between $C^{*}$-algebras is already completely bounded with $\|\phi\|_{c b}=\|\phi\|$. Unfortunately, this is not true for completely positive $k$-linear operators when $k \geq 2$ (see [4], page 155).

DEFINITION 1.1. A completely bounded and completely positive $k$ linear operator $\phi \in C B^{+}\left(A^{k}, B\right)$ is pure if, for every $\psi \in C B^{+}\left(A^{k}, B\right)$, $\psi \leq \phi$ implies $\psi=\lambda \phi$ for some $\lambda \geq 0$.

From the above definition, $\phi \in C B^{+}\left(A^{k}, B\right)$ is pure if and only if the ray $R_{\phi}=\{\lambda \phi: \lambda \geq 0\}$ determined by $\phi$ is an extreme ray in $C B^{+}\left(A^{k}, B\right)$ (cf. G. Choquet [2], Volume II).

Now we consider $B=B(H)$, the algebra of all bounded linear operators on a Hilbert space $H$. If $k=1$ and $B(H)=C$, it is well known that every pure element in $C B^{+}\left(A^{k}, C\right)=\left(A^{*}\right)^{+}$is just a positive linear functional on $A$ whose GNS representation is irreducible 
(cf. Takesaki [17] Chapter I). The pure elements in $C B^{+}(A, B(H))$ were studied by Arveson [1].

In this paper, we study the structure of pure elements in $C B^{+}\left(A^{k}, B(H)\right)$ for $k=2 l$. In particular, we give a detailed discussion for pure completely bounded and completely positive bilinear operators from $A^{2}$ into the matrix algebra $M_{n}(C)$. Applying [4], Theorem 4.1, we show, in $\S 2$, a representation theorem for pure completely bounded and completely positive $k$-linear operators from $A^{k}$ into $B(H)$. We show, in Theorem 3.2 and Theorem 3.3, that a completely bounded and completely positive bilinear operator $\phi \in$ $C B^{+}\left(A^{2}, M_{n}(C)\right)$ is pure if and only if there are bounded linear functionals $f_{1}, \ldots, f_{n}$ on $A$ such that $\phi=F^{*} \odot F$, where

$$
F=\left[\begin{array}{ccc}
f_{1} & \cdots & f_{n} \\
0 & \cdots & 0 \\
. & \cdots & . \\
0 & \cdots & 0
\end{array}\right] .
$$

Such a linear operator $F$ is unique up to multiplication by a complex number of module one. We generalize Theorem 3.2 and Theorem 3.3 to completely bounded and completely positive $2 l$-linear operators from $A^{2 l}$ into $M_{n}(C)$ in Theorem 3.4. In $\S 4$, we discuss the normal version of the above results. In $\S 5$, we apply the results in $\S 3$ (resp. in $\S 4)$ to study the pure elements in multivariable Fourier-Stieltjes algebras (resp. in multivariable Fourier algebras).

To close this section, we state a result of [4].

TheOREm 1.2 ([4], Lemma 3.1 and Corollary 4.2). Let $H$ be a Hilbert space, let $A$ be a $C^{*}$-algebra, and let $\phi \in C B_{s}\left(A^{k}, B(H)\right)$ with $k \geq 2$. Let $\varphi: A \rightarrow B(H)$ be a completely positive linear operator given by $\varphi=V^{*} \pi V$, where $\pi$ is a *-representation of $A$ on a Hilbert space $K$ and $V \in B(H, K)$ is a bounded linear operator with $K=$ $[\pi(A) V H]$. If we have

$$
-\varphi_{n}\left(X^{*} X\right) \leq \phi_{n}\left(X^{*}, A_{2}, \ldots, A_{k-1}, X\right) \leq \varphi_{n}\left(X^{*} X\right)
$$

for all $X \in M_{n}(A)$ and all $\left(A_{2}, \ldots, A_{k-1}\right)=\left(A_{k-1}^{*}, \ldots, A_{2}^{*}\right) \in$ $M_{n}(A)^{k-2}$ with $\left\|A_{j}\right\| \leq 1 \quad(2 \leq j \leq k-1)$, then there is a $\psi \in$ $C B_{s}\left(A^{k-2}, B(K)\right)$ with $\|\psi\|_{c b} \leq 1$ (when $k=2, \psi$ is just a fixed selfadjoint linear operator in $B(K))$ such that

$$
\phi\left(a_{1}, \ldots, a_{k}\right)=V^{*} \pi\left(a_{1}\right) \psi\left(a_{2}, \ldots, a_{k-1}\right) \pi\left(a_{k}\right) V
$$


for all $a_{1}, \ldots, a_{k} \in A$. If, in addition, $\phi$ is completely positive, then so is $\psi$.

REMARK. In Theorem 1.2, we considered a given representation $\{\pi, V, K\}$ of $\varphi$ with $K=[\pi(A) V H]$ the norm closure of $\pi(A) V H$, which is called a minimal representation of $\varphi$ in [1]. If $A$ is a unital $C^{*}$-algebra, the representation of $\varphi$ obtained from the Stinespring construction (cf. [1] and [16]) is minimal. Since any two minimal representations of $\varphi$ are unitarily equivalent, the result in Theorem 1.2 is essentially the same as that in [4] Lemma 3.1 .

2. A representation theorem for pure completely bounded and completely positive $k$-linear operators. Let $A$ be a $C^{*}$-algebra, let $K$ and $H$ be Hilbert spaces, let $\pi$ be a ${ }^{*}$-representation of $A$ on $K$ and let $V \in B(H, K)$ with $K=[\pi(A) V H]$. For any integer $k \geq 2$, we can define a map $\Gamma: C B\left(A^{k-2}, B(K)\right) \rightarrow C B\left(A^{k}, B(H)\right)$ by

$$
\Gamma(\psi)\left(a_{1}, \ldots, a_{k}\right)=V^{*} \pi\left(a_{1}\right) \psi\left(a_{2}, \ldots, a_{k-1}\right) \pi\left(a_{k}\right) V
$$

for all $a_{1}, \ldots, a_{k} \in A$. Here we denote $B(K)=C B\left(A^{0}, B(K)\right)$. It is clear that $\Gamma$ is a well-defined bounded linear operator from $C B\left(A^{k-2}, B(K)\right)$ into $C B\left(A^{k}, B(H)\right)$, which maps $C B_{s}\left(A^{k-2}, B(K)\right)$ into $C B_{s}\left(A^{k}, B(H)\right)$ and $C B^{+}\left(A^{k-2}, B(K)\right)$ into $C B^{+}\left(A^{k}, B(H)\right)$. It follows from $K=[\pi(A) V H]$ that $\Gamma$ is a linear injection. If $V \in B(H, K)$ is a contraction then so is $\Gamma$.

LEMMA 2.1. The linear operator $\Gamma$ is a linear order isomorphism from $C B_{s}\left(A^{k-2}, B(K)\right)$ onto its image $\Gamma\left(C B_{s}\left(A^{k-2}, B(K)\right)\right)$.

Proof. We only need to show that, for any $\phi \in C B^{+}\left(A^{k}, B(H)\right) \cap$ $\Gamma\left(C B_{s}\left(A^{K-2}, B(K)\right)\right)$, there is a $\psi \in C B^{+}\left(A^{K-2}, B(K)\right)$ such that $\Gamma(\psi)=\phi$.

Given $\phi \in C B^{+}\left(A^{k}, B(H)\right) \cap \Gamma\left(C B_{s}\left(A^{k-2}, B(K)\right)\right)$, there is a $\psi \in$ $C B_{s}\left(A^{k-2}, B(K)\right)$ such that $\Gamma(\psi)=\phi$. For each $n \in N$, and all $\left(A_{2}, \ldots, A_{k-1}\right)=\left(A_{k-1}^{*}, \ldots, A_{2}^{*}\right) \in M_{n}(A)^{k-2}$ with $A_{m} \geq 0$ if $k$ is odd, where $m=(k+1) / 2$, and for all $\eta_{n}=\pi_{n}(X)\left(V \otimes \mathrm{id}_{n}\right) \xi_{n}$, when $X \in M_{n}(A)$ and $\xi_{n} \in H^{n}$, we have

$$
\begin{aligned}
& \left\langle\psi_{n}\left(A_{2}, \ldots, A_{k-1}\right) \eta_{n}, \eta_{n}\right\rangle \\
& \quad=\left\langle\left(V^{*} \otimes \mathrm{id}_{n}\right) \pi_{n}\left(X^{*}\right) \psi_{n}\left(A_{2}, \ldots, A_{k-1}\right) \pi_{n}(X)\left(V \otimes \mathrm{id}_{n}\right) \xi_{n}, \xi_{n}\right\rangle \\
& \quad=\left\langle\phi_{n}\left(X^{*}, A_{2}, \ldots, A_{k-1}, X\right) \xi_{n}, \xi_{n}\right\rangle \geq 0 .
\end{aligned}
$$

Thus $\psi \in C B^{+}\left(A^{k-2}, B(K)\right)$ since $\pi(A) V H$ is dense in $K$. 
Let $\phi \in C B^{+}\left(A^{k}, B\right)$. The order interval $[0, \phi]$ is defined by

$$
[0, \phi]=\left\{\hat{\phi} \in C B^{+}\left(A^{k}, B\right): 0 \leq \hat{\phi} \leq \phi\right\} .
$$

LEMMA 2.2. If $\phi=\Gamma(\psi)$ for some $\psi \in C B^{+}\left(A^{k-2}, B(K)\right)$, then the order interval $[0, \phi]$ is contained in $\Gamma\left(C B^{+}\left(A^{k-2}, B(K)\right)\right.$ and $\Gamma$ is an affine isomorphism from $[0, \psi]$ onto $[0, \phi]$.

Proof. Without loss of generality, we assume that $\phi=\Gamma(\psi)$ for some $\psi \in C B^{+}\left(A^{k-2}, B(K)\right)$ with $\|\psi\|_{c b}=1$. Let $\varphi=V^{*} \pi V$. Since

$$
-I \leq \psi_{n}\left(A_{2}, \ldots, A_{k-1}\right) \leq I,
$$

we have

$$
-\varphi_{n}\left(X^{*} X\right) \leq \phi_{n}\left(X^{*}, A_{2}, \ldots, A_{k-1}, X\right) \leq \varphi_{n}\left(X^{*} X\right)
$$

for all $X \in M_{n}(A)$ and $\left(A_{2}, \ldots, A_{k-1}\right)=\left(A_{k-1}^{*}, \ldots, A_{2}^{*}\right) \in M_{n}(A)^{k-2}$ with $\left\|A_{j}\right\| \leq 1 \quad(2 \leq j \leq k-1)$. We need to show that, for any $\hat{\phi} \in[0, \phi]$, there is $\hat{\psi} \in[0, \psi]$ such that $\Gamma(\hat{\psi})=\hat{\phi}$.

Given $\hat{\phi} \in[0, \phi]$, we claim that

$$
-\phi_{n}\left(X^{*} X\right) \leq \hat{\phi}_{n}\left(X^{*}, A_{2}, \ldots, A_{k-1}, X\right) \leq \phi_{n}\left(X^{*} X\right)
$$

for all $X \in M_{n}(A)$ and $\left(A_{2}, \ldots, A_{k-1}\right)=\left(A_{k-1}^{*}, \ldots, A_{2}^{*}\right) \in M_{n}(A)^{k-2}$ with $\left\|A_{j}\right\| \leq 1 \quad(2 \leq j \leq k-1)$, and all $n \in N$.

To see this, if $k=2 l+1$, we have

$$
\left(A_{2}, \ldots, A_{k-1}\right)=\left(B_{2}^{*}, \ldots, B_{l}^{*}, B_{l+1}, B_{l}, \ldots, B_{1}\right)
$$

for some $B_{j} \in M_{n}(A)$ with $\left\|B_{j}\right\| \leq 1 \quad(j=2, \ldots, l+1)$ and $B_{l+1}$ selfadjoint. Hence we can write $B_{l+1}=B_{l+1}^{+}-B_{l+1}^{-}$, where $B_{l+1}^{+}$and $B_{l+1}^{-}$are positive in $M_{n}(A)$ with the norms less than or equal to 1 . Since

$$
\begin{aligned}
0 & \leq \hat{\phi}_{n}\left(X^{*}, B_{2}^{*}, \ldots, B_{l}^{*}, B_{l+1}^{ \pm}, B_{l}, \ldots, B_{2}, X\right) \\
& \leq \phi_{n}\left(X^{*}, B_{2}^{*}, \ldots, B_{l}^{*}, B_{l+1}^{ \pm}, B_{l}, \ldots, B_{2}, X\right) \\
& \leq \phi_{n}\left(X^{*} X\right)
\end{aligned}
$$

we have

$$
-\phi_{n}\left(X^{*} X\right) \leq \hat{\phi}_{n}\left(X^{*}, B_{2}^{*}, \ldots, B_{l}^{*}, B_{l+1}^{*}, B_{l}, \ldots, B_{2}, X\right) \leq \phi_{n}\left(X^{*} X\right) .
$$


If $k=2 l$, we have

$$
\left(A_{2}, \ldots, A_{k-1}\right)=\left(B_{2}^{*}, \ldots, B_{l}^{*}, B_{l}, \ldots, B_{2}\right)
$$

for some $B_{j} \in M_{n}(A)$ with $\left\|B_{j}\right\| \leq 1 \quad(2 \leq j \leq l)$; then we have

$$
\begin{aligned}
-\phi_{n}\left(X^{*} X\right) \leq 0 & \leq \hat{\phi}_{n}\left(X^{*}, B_{2}^{*}, \ldots, B_{l}^{*}, B_{l}, \ldots, B_{2}, X\right) \\
& \leq \phi_{n}\left(X^{*}, B_{2}^{*}, \ldots, B_{l}^{*}, B_{l}, \ldots, B_{2}, X\right) \\
& \leq \phi_{n}\left(X^{*} X\right) .
\end{aligned}
$$

By Theorem 1.2, there exists a completely bounded and completely positive $(k-2)$-linear operator $\hat{\psi} \in C B^{+}\left(A^{k-2}, B(K)\right)$ such that $\Gamma(\hat{\psi})$ $=V^{*} \pi \hat{\psi} \pi V=\hat{\phi}$. Since $\hat{\phi} \leq \phi$ and, by Lemma $2.1, \Gamma$ is a linear order isomorphism from $C B_{s}\left(A^{k-2}, B(K)\right)$ onto $\Gamma\left(C B_{s}\left(A^{k-2}, B(K)\right)\right)$, we have $\hat{\psi} \in[0, \psi]$.

LEMMA 2.3. A completely bounded and completely positive $k$-linear operator $\psi \in C B^{+}\left(A^{k-2}, B(K)\right)$ is pure if and only if its image $\phi=$ $\Gamma(\psi)$ is pure in $C B^{+}\left(A^{k}, B(H)\right)$.

Proof. From the definition, we know that $\psi$ (resp. $\phi$ ) is pure if and only if $[0, \psi]=\{\lambda \psi: 0 \leq \lambda \leq 1\}$ (resp. $[0, \phi]=\{\lambda \phi ; 0 \leq \lambda \leq 1\})$. The conclusion follows easily from Lemma 2.2

THeORem 2.4. Let $A$ be a $C^{*}$-algebra, let $H$ be a Hilbert space and let $\phi \in C B^{+}\left(A^{k}, B(H)\right)$ with $k \geq 2$. Then $\phi$ is pure if and only if

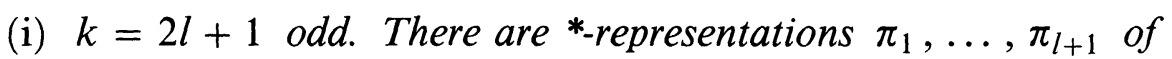
$A$ on Hilbert spaces $K_{1}, \ldots, K_{l+1}$ with $\pi_{l+1}$ irreducible on $K_{l+1}$ and linear operators $V_{j} \in B\left(K_{j-1}, K_{j}\right)$ for $1 \leq j \leq l+1$, where $K_{0}=H$, $K_{j}=\left[\pi_{j}(A) V_{j} K_{j-1}\right]$ for all $1 \leq j \leq l+1$ and

$$
\left\|V_{1}\right\| \cdots\left\|V_{l+1}\right\|=\|\phi\|_{c b}^{1 / 2}
$$

such that

$$
\begin{aligned}
& \phi\left(a_{1}, \ldots, a_{k}\right) \\
& \quad=V_{1}^{*} \pi_{1}\left(a_{1}\right) \cdots \pi_{l}\left(a_{l}\right) V_{l+1}^{*} \pi_{l+1}\left(a_{l+1}\right) V_{l+1} \pi_{l}\left(a_{l+2}\right) \cdots \pi_{1}\left(a_{k}\right) V_{1}
\end{aligned}
$$

for all $a_{1}, \ldots, a_{k} \in A$.

(ii) $k=2 l$ even. There are $*_{- \text {representations }} \pi_{1}, \ldots, \pi_{l}$ of $A$ on Hilbert spaces $K_{1}, \ldots, K_{l}$, linear operators $V_{j} \in B\left(K_{j-1}, K_{j}\right)$, where $K_{0}=H, K_{j}=\left[\pi_{j}(A) V_{j} K_{j-1}\right]$ for $1 \leq j \leq l$ and

$$
\left\|V_{1}\right\| \cdots\left\|V_{l}\right\|=\|\phi\|_{c b}^{1 / 2}
$$


and a rank one projection $T$ in $B\left(K_{l}\right)^{+}$such that

$$
\begin{aligned}
& \phi\left(a_{1}, \ldots, a_{k}\right) \\
& \quad=V_{1}^{*} \pi\left(a_{1}\right) V_{2}^{*} \pi_{2}\left(a_{2}\right) \cdots \pi_{l}\left(a_{l}\right) T \pi_{l}\left(a_{l+1}\right) \cdots \pi_{2}\left(a_{k-1}\right) V_{2} \pi_{1}\left(a_{k}\right) V_{1}
\end{aligned}
$$

for all $a_{1}, \ldots, a_{k} \in A$.

Proof. Applying [4], Theorem 4.1, we have

(i) $k=2 l+1$ odd. There are ${ }^{*}$-representations $\pi_{1}, \ldots, \pi_{l+1}$ of $A$ on Hilbert spaces $K_{1}, \ldots, K_{l+1}$ and linear operators $V_{j} \in$ $B\left(K_{j-1}, K_{j}\right)$, where $K_{0}=H, K_{j}=\left[\pi_{j}(A) V_{j} K_{j-1}\right]$ for all $1 \leq j \leq$ $l+1$ and

$$
\left\|V_{1}\right\| \cdots\left\|V_{l+1}\right\|=\|\phi\|_{c b}^{1 / 2}
$$

such that

$$
\begin{aligned}
& \phi\left(a_{1}, \ldots, a_{k}\right) \\
& \quad=V_{1}^{*} \pi_{1}\left(a_{1}\right) \cdots \pi_{l}\left(a_{l}\right) V_{l+1}^{*} \pi_{l+1}\left(a_{l+1}\right) V_{l+1} \pi_{l}\left(a_{l+2}\right) \cdots \pi_{1}\left(a_{k}\right) V_{1}
\end{aligned}
$$

for all $a_{1}, \ldots, a_{k} \in A$. By Lemma 2.3 and induction on $l$, we have $\phi \in C B^{+}\left(A^{k}, B(H)\right)$ is pure if and only if $\psi=V_{l+1}^{*} \pi_{l+1} V_{l+1}$ is pure in $C B^{+}\left(A, B\left(K_{l}\right)\right)$ if and only if $\pi$ is an irreducible representation on $K_{l+1}$ (see Arveson [1]).

(ii) $k=2 l$ even. There are ${ }^{*}$-representations $\pi_{1}, \ldots, \pi_{l}$ of $A$ on Hilbert spaces $K_{1}, \ldots, K_{l}$, linear operators $V_{j} \in B\left(K_{j-1}, K_{j}\right)$, where $K_{0}=H, K_{j}=\left[\pi_{j}(A) V_{j} K_{j-1}\right]$ for $1 \leq j \leq l$ and

$$
\left\|V_{1}\right\| \cdots\left\|V_{l}\right\|=\|\phi\|_{c b}^{1 / 2}
$$

and a positive linear operator $T \in B\left(K_{l}\right)^{+}$with $\|T\|=1$ such that

$$
\begin{aligned}
& \phi\left(a_{1}, \ldots, a_{k}\right) \\
& \quad=V_{1}^{*} \pi\left(a_{1}\right) V_{2}^{*} \pi_{2}\left(a_{2}\right) \cdots \pi_{l}\left(a_{l}\right) T \pi_{l}\left(a_{l+1}\right) \cdots \pi_{2}\left(a_{k-1}\right) V_{2} \pi_{1}\left(a_{k}\right) V_{1}
\end{aligned}
$$

for all $a_{1}, \ldots, a_{k} \in A$. By Lemma 2.3 and induction on $l$, we have $\phi \in C B^{+}\left(A^{k}, B(H)\right)$ is pure if and only if $T$ is pure in $B\left(K_{l}\right)^{+}$, the set of all positive linear operators on $K_{l}$, with $\|T\|=1$ if and only if $T$ is a rank one projection.

3. The structure of pure completely bounded and completely positive multlinear operators. In this section, we study the structure of pure completely bounded and completely positive $2 l$-linear operators from $A^{2 l}$ into $M_{n}(C)$. Let $f$ and $g$ be $l$-linear functionals from $A^{l}$ into $C$. We define a $2 l$-linear function $f \otimes g: A^{2 l} \rightarrow C$ by

$$
(f \otimes g)\left(a_{1}, \ldots, a_{2 l}\right)=f\left(a_{1}, \ldots, a_{l}\right) g\left(a_{l+1}, \ldots, a_{2 l}\right)
$$


for all $a_{1}, \ldots, a_{2 l} \in A$. Let $F=\left[f_{i j}\right]$ and $G=\left[g_{i j}\right]$ be $l$-linear operators from $A^{l}$ into $M_{n}(C)$. Then we can define a $2 l$-linear operator $F \cdot G: A^{2 l} \rightarrow M_{n}(C)$ by

$$
F \odot G=\left[\sum_{k=1}^{n} f_{i k} \otimes g_{k j}\right] .
$$

For an $l$-linear operator $F=\left[f_{i j}\right]: A^{l} \rightarrow M_{n}(C)$, the adjoint $l$ linear operator $F^{*}$ of $F$ can be written as $F^{*}=\left[f_{j i}^{*}\right]$. The $l$ linear operator $F$ is completely bounded if and only if each $f_{i j}$ is a completely bounded $l$-linear functional on $A^{l}$. If $F$ is completely bounded, then so is $F^{*}$ with $\left\|F^{*}\right\|_{c b}=\|F\|_{c b}$.

Let $F=\left[f_{i j}\right]: A^{l} \rightarrow M_{n}(C)$ be a completely bounded $l$-linear operator. For all $A_{j}=\left[a_{i_{j} i_{j+1}}\right] \in M_{m}(A) \quad(1 \leq j \leq 2 l)$, we have

$$
\begin{aligned}
\left(F^{*} \odot\right. & F)_{m}\left(A_{1}, \ldots, A_{2 l}\right) \\
= & {\left[\sum_{i_{2}, \ldots, i_{2 l}=1}^{m}\left(F^{*} \odot F\right)\left(a_{i_{1} i_{2}}, \ldots, a_{i_{2 l} i_{2 l+1}}\right)\right] } \\
= & {\left[\sum_{i_{l+1}=1}^{m}\left(\sum_{i_{2}, \ldots, i_{l}=1}^{m} F^{*}\left(a_{i_{1} i_{2}}, \ldots, a_{i_{l} i_{l+1}}\right)\right)\right.} \\
& \left.\cdot\left(\sum_{i_{l+2}, \ldots, i_{2 l}}^{m} F\left(a_{i_{l+1} i_{l+2}}, \ldots, a_{i_{2 l} i_{2 l+1}}\right)\right)\right] \\
= & F_{m}^{*}\left(A_{1}, \ldots, A_{l}\right) F_{m}\left(A_{l+1}, \ldots, A_{2 l}\right)
\end{aligned}
$$

in $M_{m}(C) \otimes M_{n}(C)$. Since

$$
F_{m}^{*}\left(A_{1}^{*}, \ldots, A_{l}^{*}\right)=\left(F_{m}\left(A_{l}, \ldots, A_{1}\right)\right)^{*}
$$

for all $A_{1}, \ldots, A_{l} \in M_{m}(A)$, we have

$$
\begin{aligned}
& \left(F^{*} \odot F\right)_{m}\left(A_{1}^{*}, \ldots, A_{l}^{*}, A_{l}, \ldots, A_{1}\right) \\
& \quad=F_{m}^{*}\left(A_{1}^{*}, \ldots, A_{l}^{*}\right) F_{m}\left(A_{l}, \ldots, A_{1}\right) \\
& \quad=\left(F_{m}\left(A_{l}, \ldots, A_{1}\right)\right)^{*} F_{m}\left(A_{l}, \ldots, A_{1}\right) .
\end{aligned}
$$

This implies that $F^{*} \odot F: A^{2 l} \rightarrow M_{n}(C)$ is completely positive.

LEMMA 3.1. Let $F=\left[f_{i j}\right]: A^{l} \rightarrow M_{n}(C)$ be an l-linear operator. Then the corresponding 2l-linear operator $\phi=F^{*} \odot F: A^{2 l} \rightarrow M_{n}(C)$ is completely positive. The l-linear operator $F$ is completely bounded 
if and only if the 2l-linear operator $\phi=F^{*} \odot F$ is completely bounded. In this case, we have $\|\phi\|_{c b}=\left\|\phi_{n}\right\|=\|F\|_{c b}^{2}$.

Proof. The first statement is obvious. For the rest of the proof, we consider the case $l=1$ without loss of the generality. If $F$ is completely bounded, we have

$$
\begin{aligned}
\|\phi\|_{c b} & =\sup \left\{\left\|\phi_{m}(X, Y)\right\|: X, Y \in M_{m}(A)\right. \\
& \quad \text { with }\|X\|,\|Y\| \leq 1, m \in N\} \\
& =\sup \left\{\left\|F_{m}^{*}(X) F_{m}(Y)\right\|: X, Y \in M_{m}(A)\right. \\
& \quad \text { with }\|X\|,\|Y\| \leq 1, m \in N\} \\
& \leq\left\|F^{*}\right\|_{c b}\|F\|_{c b}=\|F\|_{c b}^{2} .
\end{aligned}
$$

Hence $\phi$ is completely bounded. On the other hand, if $\phi$ is completely bounded, we have

$$
\begin{aligned}
\|F\|_{c b}^{2} & =\left\|F_{n}\right\|^{2} \quad(\text { by [15]) } \\
& =\sup \left\{\left\|F_{n}(X)\right\|^{2}: X \in M_{n}(A),\|X\| \leq 1\right\} \\
& =\sup \left\{\left\|\left(F_{n}(X)\right)^{*} F_{n}(X)\right\|: X \in M_{n}(A),\|X\| \leq 1\right\} \\
& =\sup \left\{\left\|\phi_{n}\left(X^{*}, X\right)\right\|: X \in M_{n}(A),\|X\| \leq 1\right\} \\
& \leq\left\|\phi_{n}\right\| \leq\|\phi\|_{c b} .
\end{aligned}
$$

Hence $F$ is completely bounded and we have $\|F\|_{c b}^{2}=\left\|F_{n}\right\|^{2}=$ $\left\|\phi_{n}\right\|=\|\phi\|_{c b}$.

Now we are ready to study the structure of pure completely bounded and completely positive multilinear operators. For our convenience, we first consider the bilinear case.

THEOREM 3.2. If $\phi \in C B^{+}\left(A^{2}, M_{n}(C)\right)$ is pure, then there are bounded linear functionals $f_{1}, \ldots, f_{n}$ on $A$ such that $\phi=F^{*} \odot F$, where

$$
F=\left[\begin{array}{ccc}
f_{1} & \cdots & f_{n} \\
0 & \cdots & 0 \\
. & \cdots & . \\
0 & \cdots & 0
\end{array}\right]
$$

The linear operator $F$ is completely bounded with $\|F\|_{c b}^{2}=\|\phi\|_{c b}$.

Furthermore, if

$$
G=\left[\begin{array}{ccc}
g_{1} & \cdots & g_{n} \\
0 & \cdots & 0 \\
. & \cdots & . \\
0 & \cdots & 0
\end{array}\right]
$$


is another completely bounded linear operator from $A$ into $M_{n}(C)$ such that $\phi=G^{*} \odot G$, then there is a complex number $\lambda$ with $|\lambda|=1$ such that $F=\lambda G$.

Proof. To avoid technical complications, we only discuss the case $n=2$. The calculations are in the same spirit for general $n \in N$.

Let $\phi \neq 0 \in C B^{+}\left(A^{2}, M_{2}(C)\right)$ be a pure element. By Theorem 2.4, there is a ${ }^{*}$-representation $\pi$ of $A$ on a Hilbert space $K$, a bounded linear operator $V: C^{2} \rightarrow K$ with $K=\left[\pi(A) a V C^{2}\right]$ and $\|V\|=\|\phi\|_{c b}^{1 / 2}$ and a rank one projection $T$ in $B(K)$ such that

$$
\phi(x, y)=V^{*} \pi(x) T \pi(y) V
$$

for all $x, y \in A$. Let $\xi_{0}$ be a unit vector in $K$ such that $T K=$ $\left[T \pi(A) V C^{2}\right]=\operatorname{span}\left\{\xi_{0}\right\}$ and let $\left\{e_{1}=\left[\begin{array}{l}1 \\ 0\end{array}\right], e_{2}=\left[\begin{array}{l}0 \\ 1\end{array}\right]\right\}$ be the standard basis for $C^{2}$. For $i=1,2$, there are linear functionals $f_{i}$ on $A$ such that

$$
f_{i}(x) \xi_{0}=T \pi(x) V e_{i}
$$

for all $x \in A$. Then we have

$$
\begin{aligned}
& \left\langle\phi(x, y)\left[\begin{array}{l}
\alpha_{1} \\
\alpha_{2}
\end{array}\right],\left[\begin{array}{l}
\beta_{1} \\
\beta_{2}
\end{array}\right]\right\rangle=\sum_{i, j=1}^{2} \bar{\beta}_{i} \alpha_{j}\left\langle\phi(x, y) e_{j}, e_{i}\right\rangle \\
& =\sum_{i, j=1}^{2} \bar{\beta}_{i} \alpha_{j}\left\langle T \pi(y) V e_{j}, T \pi\left(x^{*}\right) V e_{i}\right\rangle \\
& =\sum_{i, j=1}^{2} \bar{\beta}_{i} \alpha_{j}\left\langle f_{j}(y) \xi_{0}, f_{i}\left(x^{*}\right) \xi_{0}\right\rangle \\
& =\sum_{i, j=1}^{2} \bar{\beta}_{i} \alpha_{j} f_{j}(y) \overline{f_{i}\left(x^{*}\right)} \\
& =\left\langle\left[\begin{array}{ll}
f_{1}^{*}(x) & 0 \\
f_{2}^{*}(x) & 0
\end{array}\right]\left[\begin{array}{cc}
f_{1}(y) & f_{2}(y) \\
0 & 0
\end{array}\right]\left[\begin{array}{l}
\alpha_{1} \\
\alpha_{2}
\end{array}\right],\left[\begin{array}{l}
\beta_{1} \\
\beta_{2}
\end{array}\right]\right\rangle
\end{aligned}
$$

for all $x, y \in A$ and for all $\alpha_{i}, \beta_{i} \in C \quad(i=1,2)$. This implies that $\phi=F^{*} \odot F$, where $F=\left[\begin{array}{cc}f_{1} & f_{2} \\ 0 & 0\end{array}\right]$. It follows from Lemma 3.1 that $F: A \rightarrow M_{2}(C)$ is completely bounded with $\|F\|_{c b}^{2}=\|\phi\|_{c b}$.

Suppose that $G=\left[\begin{array}{cc}g_{1} & g_{2} \\ 0 & 0\end{array}\right]: A \rightarrow M_{2}(C)$ is another completely bounded linear operator such that $\phi=G^{*} \odot G$. Since $\left\|F^{*}\right\|_{c b}=\|F\|_{c b}=$ $\|\phi\|_{c b}^{1 / 2} \neq 0$, we may assume that $f_{1}^{*} \neq 0$. Then there is an element 
$x_{0} \in A$ such that $f_{1}^{*}\left(x_{0}\right) \neq 0$. Thus for all $x \in A$, we have

$$
\begin{aligned}
{\left[f_{i}^{*}\left(x_{0}\right) f_{j}(x)\right] } & =\left(F^{*} \odot F\right)\left(x_{0}, x\right)=\phi\left(x_{0}, x\right) \\
& =\left(G^{*} \odot G\right)\left(x_{0}, x\right)=\left[g_{i}^{*}\left(x_{0}\right) g_{j}(x)\right] .
\end{aligned}
$$

If we let $\lambda=g_{1}^{*}\left(x_{0}\right) / f_{1}^{*}\left(x_{0}\right)$, then we get $f_{j}(x)=\lambda g_{j}(x)$ for all $x \in A$ $(j=1,2)$, i.e. we get $F=\lambda G$. Since $\|G\|_{c b}=\|\phi\|_{c b}^{1 / 2}=\|F\|_{c b}=$ $|\lambda|\|G\|_{c b}$, then $|\lambda|=1$.

THEOREM 3.3. Let

$$
\left[\begin{array}{ccc}
f_{1} & \cdots & f_{n} \\
0 & \cdots & 0 \\
. & \cdots & . \\
0 & \cdots & 0
\end{array}\right]: A \rightarrow M_{n}(C)
$$

be a completely bounded linear operator. Then $\phi=F^{*} \odot F$ is a pure completely bounded and completely positive bilinear operator in $C B^{+}\left(A^{2}, M_{n}(C)\right)$ with $\|\phi\|_{c b}=\|F\|_{c b}^{2}$.

Proof. It follows, from Lemma 3.1, that $\phi$ is a completely bounded and completely positive bilinear operator from $A$ into $M_{n}(C)$ with $\|\phi\|_{c b}=\|F\|_{c b}^{2}$. It suffices to show that $\phi$ is pure in $C B^{+}\left(A^{2}, M_{n}(C)\right)$. Here we only prove the case $n=2$ as in Theorem 3.2.

Since $\phi=F^{*} \odot F \in C B^{+}\left(A^{2}, M_{2}(C)\right)$, by [4] Theorem 4.1, there is a ${ }^{*}$-representation $\pi$ of $A$ on a Hilbert space $K$, a bounded linear operator $V: C^{2} \rightarrow K$ with $K=\left[\pi(A) V C^{2}\right]$ and $\|V\|=\|\phi\|_{c b}^{1 / 2}$, and a positive linear operator $T$ in $B(K)$ with $\|T\|=1$ such that

$$
\phi(x, y)=V^{*} \pi(x) T \pi(y) V
$$

for all $x, y \in A$. From Theorem 2.4 , it suffices to show that $T$ is a rank one projection.

Since very element $\eta \in \pi(A) V C^{2}$ can be written as

$$
\eta=\sum_{i=1}^{2} \pi\left(x_{i}\right) V e_{i}
$$

for some $x_{i} \in A \quad(i=1,2)$, we define a linear functional $\hat{f}: \pi(A) V C^{2}$ $\rightarrow C$ by

$$
\hat{f}\left(\sum_{i=1}^{2} \pi\left(x_{i}\right) V e_{i}\right)=\sum_{i=1}^{2} f_{i}\left(x_{i}\right)
$$


for all $\sum_{i=1}^{2} \pi\left(x_{i}\right) V e_{i} \in \pi(A) V C^{2}$. Since

$$
\begin{aligned}
&\left|\sum_{i=1}^{2} f_{i}\left(x_{i}\right)\right|^{2}=\sum_{i, j=1}^{2} \overline{f_{i}\left(x_{i}\right)} f_{j}\left(x_{j}\right) \\
&=\sum_{i, j=1}^{2} f_{i}^{*}\left(x_{i}^{*}\right) f_{j}\left(x_{j}\right) \\
&=\left\langle\left[\begin{array}{ll}
F^{*}\left(x_{1}^{*}\right) & 0 \\
F^{*}\left(x_{2}^{*}\right) & 0
\end{array}\right]\left[\begin{array}{cc}
F\left(x_{1}\right) & F\left(x_{2}\right) \\
0 & 0
\end{array}\right]\left[\begin{array}{l}
e_{1} \\
e_{2}
\end{array}\right],\left[\begin{array}{l}
e_{1} \\
e_{2}
\end{array}\right]\right\rangle \\
&=\left\langle\phi_{2}\left(\left[\begin{array}{ll}
x_{1}^{*} & 0 \\
x_{2}^{*} & 0
\end{array}\right],\left[\begin{array}{cc}
x_{1} & x_{2} \\
0 & 0
\end{array}\right]\right)\left[\begin{array}{l}
e_{1} \\
e_{2}
\end{array}\right],\left[\begin{array}{l}
e_{1} \\
e_{2}
\end{array}\right]\right\rangle \\
&=\left\langle\left(T \otimes \mathrm{id}_{2}\right) \pi_{2}\left(\left[\begin{array}{cc}
x_{1} & x_{2} \\
0 & 0
\end{array}\right]\right)\left(V \otimes \mathrm{id}_{2}\right)\left[\begin{array}{l}
e_{1} \\
e_{2}
\end{array}\right],\right. \\
&\left.\pi_{2}\left(\left[\begin{array}{cc}
x_{1} & x_{2} \\
0 & 0
\end{array}\right]\right)\left(V \mathrm{id}_{2}\right)\left[\begin{array}{l}
e_{1} \\
e_{2}
\end{array}\right]\right\rangle \\
&=\left\langle T \sum_{i=1}^{2} \pi\left(x_{i}\right) V e_{i}, \sum_{i=1}^{2} \pi\left(x_{i}\right) V e_{i}\right\rangle,
\end{aligned}
$$

it follows that $\hat{f}$ is well-defined and we have

$$
\left|\hat{f}\left(\sum_{i=1}^{2} \pi\left(x_{i}\right) V e_{i}\right)\right|^{2}=\left|\sum_{i=1}^{2} f_{i}\left(x_{i}\right)\right|^{2}=\left\langle T \sum_{i=1}^{2} \tau\left(x_{i}\right) V e_{i}, \sum_{i=1}^{2} \pi\left(x_{i}\right) V e_{i}\right\rangle .
$$

Therefore,

$$
\begin{aligned}
& \|\hat{f}\|=\sup \left\{\left|\hat{f}\left(\sum_{i=1}^{2} \pi\left(x_{i}\right) V e_{i}\right)\right|:\left\|\sum_{i=1}^{2} \pi\left(x_{i}\right) V e_{i}\right\| \leq 1\right\} \\
& =\sup \left\{\left|\left\langle T \sum_{i=1}^{2} \pi\left(x_{i}\right) V e_{i}, \sum_{i=1}^{2} \pi\left(x_{i}\right) V e_{i}\right\rangle\right|^{1 / 2}\right. \text { : } \\
& \left.\left\|\sum_{i=1}^{2} \pi\left(x_{i}\right) V e_{i}\right\| \leq 1\right\} \\
& =\|T\|=1 .
\end{aligned}
$$

Since $\pi(A) V C^{2}$ is a dense subspace of $K$, there is a unique norm preserving linear extension of $\hat{f}$ from $\pi(A) V C^{2}$ to the whole Hilbert space $K=\left[\pi(A) V C^{2}\right]$, still denoted by $\hat{f}$. For $x_{i}, y_{i} \in A(i=1,2)$, 
the identities

$$
\begin{aligned}
& \overline{\hat{f}\left(\sum_{i=1}^{2} \pi\left(x_{i}\right) V e_{i}\right)} \hat{f}\left(\sum_{j=1}^{2} \pi\left(y_{j}\right) V e_{j}\right) \\
& =\left(\sum_{i=1}^{2} f_{i}^{*}\left(x_{i}^{*}\right)\right)\left(\sum_{j=1}^{2} f_{j}\left(y_{j}\right)\right)=\left\langle T \sum_{j=1}^{2} \pi\left(y_{j}\right) V e_{j}, \sum_{i=1}^{2} \pi\left(x_{i}\right) V e_{i}\right\rangle
\end{aligned}
$$

lead to

$$
\hat{f}(\eta) \overline{\hat{f}(\zeta)}=\langle T \eta, \zeta\rangle
$$

for all $\eta, \zeta \in K$. Since $\|\hat{f}\|=1$, there is a unit vector $\omega_{0} \in K$ such that $\hat{f}(\eta)=\left\langle\eta, \omega_{0}\right\rangle$ for all $\eta \in K$. Let $K_{1}=\operatorname{span}\left\{\omega_{0}\right\}$. Since $(\operatorname{ker} \hat{f})=K_{1}^{\perp}$, the Hilbert space $K$ can be orthogonally decomposed into the direct sum of $K_{1}$ and $\operatorname{ker} \hat{f}$, i.e. $K=K_{1} \oplus \operatorname{ker} \hat{f}$.

Finally we want to show that $T$ is a projection from $K$ onto the one dimensional subspace $K_{1}$ of $K$. For every $\eta \in \operatorname{ker} \hat{f}$, we have $T \eta=0$ since $\langle T \eta, \zeta\rangle=\hat{f}(\eta) \overline{\hat{f}(\zeta)}=0$ for all $\zeta \in K$. Suppose that $T \omega_{0}=\alpha \omega_{0}+\eta_{0}$ for some $\alpha \in C$ and $\eta_{0} \in \operatorname{ker} \hat{f}$. It follows that

$$
\alpha=\left\langle T \omega_{0}, \omega_{0}\right\rangle=\hat{f}\left(\omega_{0}\right) \overline{\hat{f}\left(\omega_{0}\right)}=\left|\hat{f}\left(\omega_{0}\right)\right|^{2}=1
$$

and

$$
\left\|\eta_{0}\right\|^{2}=\left\langle T \omega_{0}, \eta_{0}\right\rangle=\hat{f}\left(\omega_{0}\right) \overline{\hat{f}\left(\eta_{0}\right)}=0 .
$$

Hence we have $T \omega_{0}=\omega_{0}$. This shows that $T$ is a projection from $K$ onto $K_{1}$.

Finally we generalize our results in Theorem 3.2 and Theorem 3.3 to the $2 l$-linear operators. The proof is essentially the same as those in Theorem 3.2 and Theorem 3.3.

THEOREM 3.4. Let $\phi \in C B^{+}\left(A^{2 l}, M_{n}(C)\right)$. Then $\phi$ is pure if and only if there are completely bounded l-linear functionals $f_{1}, \ldots, f_{n}$ on $A^{l}$ such that $\phi=F^{*} \odot F$, where

$$
F=\left[\begin{array}{ccc}
f_{1} & \cdots & f_{n} \\
0 & \cdots & 0 \\
. & \cdots & . \\
0 & \cdots & 0
\end{array}\right]
$$

The l-linear operator $F$ is completely bounded with $\|F\|_{c b}^{2}=\|\phi\|_{c b}$. 
Furthermore, if

$$
G=\left[\begin{array}{ccc}
g_{1} & \cdots & g_{n} \\
0 & \cdots & 0 \\
. & \cdots & . \\
0 & \cdots & 0
\end{array}\right]
$$

is another completely bounded $l$-linear operator from $A^{l}$ into $M_{n}(C)$ such that $\phi=G^{*} \odot G$, then there is a complex number $\lambda$ with $|\lambda|=1$ such that $F=\lambda G$.

4. Pure completely bounded and completely positive normal multilinear operators. If $R$ is a von Neumann algebra, a completely bounded $k$-linear operator $\phi: R^{k} \rightarrow B(H)$ is called normal if $\phi$ is $\sigma$-weakly continuous in each component (cf. [11] and [4]). We denote $C B^{\sigma}\left(R^{k}, B(H)\right)$ the space of all completely bounded normal $k$-linear operators from $R^{k}$ into $B(H)$. We write

$$
C B_{s}^{\sigma}\left(R^{k}, B(H)\right)=C B_{s}\left(R^{k}, B(H)\right) \cap C B^{\sigma}\left(R^{k}, B(H)\right)
$$

and

$$
C B^{\sigma+}\left(R^{k}, B(H)\right)=C B^{+}\left(R^{k}, B(H)\right) \cap C B^{\sigma}\left(R^{k}, B(H)\right) .
$$

In this section, we discuss the structures of pure completely bounded and completely positive normal $2 l$-linear operators from $R^{2 l}$ into $M_{n}(C)$. First we consider a normal version of Theorem 2.4.

THEOREM 4.1. Let $\phi \in C B^{\sigma+}\left(R^{2 l}, B(H)\right)$ be a completely bounded and completely positive normal 2l-linear operator $R^{2 l}$ into $B(H)$ for some Hilbert space $H$. Then $\phi$ is a pure element in $C B^{\sigma+}\left(R^{2 l}, B(H)\right)$ if and only if there are normal ${ }^{*}$-representations $\pi_{1}, \ldots, \pi_{l}$ of $R$ on Hilbert spaces $K_{1}, \ldots, K_{l}$, linear operators $V_{j} \in B\left(K_{j-1}, K_{j}\right)$, where $K_{0}=H, K_{j}=\left[\pi_{j}(R) V_{j} K_{j-1}\right]$ for $1 \leq j \leq l$ and

$$
\left\|V_{1}\right\| \cdots\left\|V_{l}\right\|=\|\phi\|_{c b}^{1 / 2}
$$

and a rank one projection $T$ in $B(K)$ such that

$$
\phi\left(a_{1}, \ldots, a_{2 l}\right)=V_{1}^{*} \pi_{1}\left(a_{1}\right) \cdots \pi_{l}\left(a_{l}\right) T \pi_{l}\left(a_{l+1}\right) \cdots \pi_{1}\left(a_{2 l}\right) V_{1}
$$

for all $a_{1}, \ldots, a_{2 l} \in R$.

Proof. Given $\phi \in C B^{\sigma+}\left(R^{2 l}, B(H)\right)$ a completely bounded and completely positive normal $2 l$-linear operator. It follows from [4], 
Theorem 4.1, and the proof of Corollary 5.7 that there are normal *representations $\pi_{1}, \ldots, \pi_{l}$ of $R$ on Hilbert spaces $K_{1}, \ldots, K_{l}$, linear operators $V_{j} \in B\left(K_{j-1}, K_{j}\right)$, where $K_{0}=H, K_{j}=\left[\pi_{j}(R) V_{j} K_{j-1}\right]$ for $1 \leq j \leq l$ and

$$
\left\|V_{1}\right\| \cdots\left\|V_{l}\right\|=\|\phi\|_{c b}^{1 / 2}
$$

and a positive linear operator $T$ in $B(K)$ with $\|T\|=1$ such that

$$
\phi\left(a_{1}, \ldots, a_{2 l}\right)=V_{1}^{*} \pi_{1}\left(a_{1}\right) \cdots \pi_{l}\left(a_{l}\right) T \pi_{l}\left(a_{l+1}\right) \cdots \pi_{1}\left(a_{2 l}\right) V_{1}
$$

for all $a_{1}, \ldots, a_{2 l} \in R$. By Lemma 2.3 and induction on $l$, we get that $\phi$ is pure if and only if $T$ is pure in $B\left(K_{l}\right)^{+}$with $\|T\|=1$ if and only if the positive linear operator $T$ is a rank one projection.

THEOREM 4.2. Let $\phi \in C B^{+}\left(R^{2 l}, M_{n}(C)\right)$ be a completely bounded and completely positive 2l-linear operator. Then $\phi$ is pure in $C B^{\sigma+}\left(R^{2 l}, M_{n}(C)\right)$ if and only if there are completely bounded normal l-linear functionals $f_{1}, \ldots, f_{n}$ on $R^{l}$ such that $\phi=F^{*} \odot F$, where

$$
F=\left[\begin{array}{ccc}
f_{1} & \cdots & f_{n} \\
0 & \cdots & 0 \\
. & \cdots & . \\
0 & \cdots & 0
\end{array}\right]
$$

The normal l-linear operator $F$ is completely bounded with $\|F\|_{c b}^{2}=$ $\|\phi\|_{c b}$.

Furthermore, if

$$
G=\left[\begin{array}{ccc}
g_{1} & \cdots & g_{n} \\
0 & \cdots & 0 \\
. & \cdots & \dot{0}
\end{array}\right]
$$

is another completely bounded normal l-linear operator from $R^{l}$ into $M_{n}(C)$ such that $\phi=G^{*} \odot G$, then there is a complex number $\lambda$ with $|\lambda|=1$ such that $F=\lambda G$.

Proof. The whole proof of this theorem is similar to those in Theorem 3.2 and Theorem 3.3. The only thing we need to point out here is that, if $\phi \in C B^{\sigma+}\left(R^{2 l}, M_{n}(C)\right)$ is pure, then there are completely bounded normal $l$-linear functionals $f_{1}, \ldots, f_{n}$ on $R^{l}$ such that $\phi=F^{*} \odot F$, where

$$
F=\left[\begin{array}{ccc}
f_{1} & \cdots & f_{n} \\
0 & \cdots & 0 \\
. & \cdots & . \\
0 & \cdots & 0
\end{array}\right]
$$


We can get $l$-linear functionals $f_{1}, \ldots, f_{n}$ normal on $R^{l}$ since we can choose normal representations $\pi_{i}=R \rightarrow B\left(K_{i}\right)(1 \leq i \leq l)$ in Theorem 4.1.

5. Application to multivariable Fourier-Stieltjes algebras and multivariable Fourier algebras. Throughout this section, we let $G$ be a discrete group, $C^{*}(G)$ the full group $C^{*}$-algebra of $G, \lambda$ the (left) regular representation of $G$ on the Hilbert space $l^{2}(G)$ and $\nu N(G)$ the group von Neumann algebra of $G$. The Fourier-Stieltjes algebra $B(G)$ is the space of all coefficients of unitary representations of $G: f \in B(G)$ if and only if there exists a unitary representation $\pi$ of $G$ on a Hilbert space $H$ and two vectors $\xi$ and $\eta$ in $H$ such that

$$
f(t)=\langle\pi(t) \xi, \eta\rangle
$$

for all $t \in G$. The norm is given by

$$
\|f\|=\inf \{\|\xi\|\|\eta\| \text {; where } \xi \text { and } \eta \text { as above }\} \text {. }
$$

It is known by Eymard [9] that $B(G)$ is a commutative Banach *algebra of functions on $G$ with the pointwise multiplication and complex conjugation, and that $B(G)$ can be identified with $C^{*}(G)^{*}$ the dual space $C^{*}(G)$ as follows:

For any $\omega \in C^{*}(G)^{*}$, we have by GNS representation

$$
\omega(a)=\langle\pi(a) \xi, \eta\rangle
$$

for all $a \in C^{*}(G)$, where $\pi$ is a *-representation of $C^{*}(G)$ on $H$ and $\xi, \eta \in H$. Thus the corresponding element $f_{\omega} \in B(G)$ can be defined by

$$
f_{\omega}(t)=\langle\pi(t), \xi, \eta\rangle
$$

for all $t \in G$.

The Fourier algebra $A(G)$ is the space of all coefficients of the (left) regular representation $\lambda$ of $G: f \in A(G)$ if and only if there exist $\xi$ and $\eta \in l^{2}(G)$ such that

$$
f(t)=\langle\lambda(t) \xi, \eta\rangle
$$

for all $t \in G$. The norm is given by

$$
\|f\|=\inf \{\|\xi\|\|\eta\| ; \text { where } \xi \text { and } \eta \text { as above }\} \text {. }
$$

Then $A(G)$ is a closed ideal of $B(G)$ and $A(G)$ is isometrically isomorphic to $\nu N(G)_{*}$, the predual of $\nu N(G)$ (cf. Eymard [9]).

The multivariable Fourier-Stieltjes algebra $B^{k}(G)$ and the multivariable Fourier algebra $A^{k}(G)$ have been studied in [8], where we 
identified $B^{k}(G)$ with $\left(C^{*}(G) \otimes_{h} \cdots \otimes_{h} C^{*}(G)\right)^{*}$ the dual space of the Haagerup tensor product of $C^{*}(G)$ 's and we identified $A^{k}(G)$ with $\left(\nu N(G) \otimes_{h}^{\sigma} \cdots \otimes_{h}^{\sigma} \nu N(G)\right)_{*}$ the predual space of the normal Haagerup tensor product of $\nu N(G)$ 's. We notice that $C^{*}(A) \otimes_{h} \cdots \otimes_{h} C^{*}(G)$ and $\nu N(G) \otimes_{h}^{\sigma} \cdots \otimes_{h}^{\sigma} \nu N(G)$ are operator space and $\sigma$-weakly closed operator space, respectively, and we denote that

$$
C B\left(C^{*}(G) \otimes_{h} \cdots \otimes_{h} C^{*}(G), B(H)\right)=C B\left(C^{*}(G)^{k}, B(H)\right)
$$

and

$$
C B^{\sigma}\left(\nu N(G) \otimes_{h}^{\sigma} \cdots \otimes_{h}^{\sigma} \nu N(G), B(H)\right)=C B^{\sigma}\left(\nu N(G)^{k}, B(H)\right) .
$$

For the detail about Haagerup tensor products, please refer to [6], [13], [14], [7] and [3].

In this section, we will restrict our attention to study the pure elements in the bi-Fourier-Stieltjes algebra $B^{2}(G)$ (resp. the bi-Fourier algebra $A^{2}(G)$ ). We recall by [8] that $f \in B^{2}(G)$ if and only if there are unitary representations $\pi_{i}$ of $G$ on Hilbert spaces $H_{i}(i=1,2)$, $T \in B\left(H_{1}, H_{2}\right)$ and $\xi \in H_{1}$ and $\eta \in H_{2}$ such that

$$
f\left(t_{2}, t_{1}\right)=\left\langle\pi_{2}\left(t_{2}\right) T \pi_{1}\left(t_{1}\right) \xi, \eta\right\rangle
$$

for all $t_{1}, t_{2} \in G$. The norm is given by

$$
\|f\|=\inf \{\|T\|\|\xi\|\|\eta\| \text {; where } T, \xi \text { and } \eta \text { as above }\} \text {. }
$$

Identifying $B^{2}(G) \quad$ (resp. $B^{2}(G)^{+}$) with $C B\left(C^{*}(G)^{2}, \mathbb{C}\right.$ ) (resp. $\left.C B^{+}\left(C^{*}(G)^{2}, \mathbb{C}\right)\right)$, there is natural order structure on $B^{2}(G)$ given by the positive cone $B^{2}(G)^{+}$with $B^{2}(G)=\operatorname{span} B^{2}(G)^{+}$. It follows easily from [4], Theorem 4.1, that $f \in B^{2}(G)^{+}$if and only if there is a unitary representation $\pi$ of $G$ on a Hilbert space $H$, a positive linear operator $T \in B(H)$ and a vector $\xi \in H$ such that

$$
f\left(t_{2}, t_{1}\right)=\left\langle\pi\left(t_{2}\right) T \pi\left(t_{1}\right) \xi, \xi\right\rangle
$$

for all $t_{1}, t_{2} \in G$. Applying Theorem 3.2 and Theorem 3.3 to the pure elements in $B^{2}(G)^{+}$, we have

THEOREM 5.1. Let $f \in B^{2}(G)$. Then $f$ is pure if and only if there is an element $g \in B(G)$ such that

$$
f=g^{*} \otimes g .
$$

We have $\|f\|=\|g\|^{2}$ and the element $g$ is unique up to multiplication by a complex number of module one. Therefore, the algebraic tensor product $B(G) \otimes B(G)=\operatorname{span}\left\{\right.$ all pure elements in $\left.B^{2}(G)^{+}\right\}$. 
REMARK. If we identify $M_{n}\left(B^{2}(G)\right)$ with $C B\left(C^{*}(G)^{2}, M_{n}(\mathbb{C})\right)$ for $n \in N$, we get an $\mathscr{L}^{\infty}$-matricial norm over $B^{2}(G)$ so that $B^{2}(G)$ is an operator space (cf. [14] and [7]). For each $n \in \mathbb{N}$, there is a natural order on $M_{n}\left(B^{2}(G)\right)$ given by the positive cone $M_{n}\left(B^{2}(G)\right)^{+}$ $=C B^{+}\left(C^{*}(G)^{2}, M_{n}(\mathbb{C})\right)$ with $M_{n}\left(B^{2}(G)\right)=\operatorname{span} M_{n}\left(B^{2}(G)\right)^{+}$. It follows from Theorem 3.2 and Theorem 3.3 that an element $\Phi \in$ $M_{n}\left(B^{2}(G)\right)^{+}$is pure if and only if there are elements $f_{1}, \ldots, f_{n} \in$ $B(G)$ such that $\Phi=F^{*} \odot F$ and $\|\phi\|=\|F\|^{2}$, where

$$
F=\left[\begin{array}{ccc}
f_{1} & \cdots & f_{n} \\
0 & \cdots & 0 \\
. & \cdots & . \\
0 & \cdots & 0
\end{array}\right] \in M_{n}(B(G))=C B\left(B(G), M_{n}(\mathbb{C})\right)
$$

Similar arguments apply to the pure elements in the bi-Fourier algebra $A^{2}(G)$. It follows by [4] and [8] that $f \in A^{2}(G)$ if and only if there are Hilbert spaces $H_{1}$ and $H_{2}$, a linear operator $T \in$ $B\left(l^{2}(G) \otimes H_{1}, l^{2}(G) \otimes H_{2}\right)$ and vectors $\xi \in l^{2}(G) \otimes H_{1}$ and $\eta \in l^{2}(G)$ $\otimes \mathrm{H}_{2}$ such that

$$
f\left(t_{2}, t_{1}\right)=\left\langle\left(\lambda\left(t_{2}\right) \otimes 1_{H_{2}}\right) T\left(\lambda\left(t_{1}\right) \otimes 1_{H_{1}}\right) \xi, \eta\right\rangle
$$

for all $t_{1}, t_{2} \in G$, and that $f \in A^{2}(A)^{+}=C B^{\sigma+}\left(\nu N(A)^{2}, \mathbb{C}\right)$ if and only if there is a Hilbert space $H$, a positive linear operator $T \in B\left(l^{2}(G) \otimes H\right)$ and $\xi \in l^{2}(G) \otimes H$ such that

$$
f\left(t_{2}, t_{1}\right)=\left\langle\left(\lambda\left(t_{2}\right) \otimes 1_{H}\right) T\left(\lambda\left(t_{1}\right) \otimes 1_{H}\right) \xi, \xi\right\rangle
$$

for all $t_{1}, t_{2} \in G$. Applying Theorem 4.2 to the pure elements in $A^{2}(G)^{+}$, we have

THEOREM 5.2. Let $f \in A^{2}(G)^{+}$. Then $f$ is pure if and only if there is an element $g \in A(G)$ such that

$$
f=g^{*} \otimes g .
$$

We have $\|f\|=\|g\|^{2}$ and the element $g$ is unique up to multiplication by a complex number of module one. Therefore the algebraic tensor product $A(G) \otimes A(G)=\operatorname{span}\left\{\right.$ all pure elements in $\left.A^{2}(G)^{+}\right\}$.

\section{REFERENCES}

[1] W. B. Arverson, Subalgebras of $C^{*}$-algebras, Acta Math., 123 (1969), 142-224.

[2] G. Choquet, Lectures on Analysis, W. A. Benjamin, Inc., 1969. 
[3] E. Christensen, E. G. Effros and A. M. Sinclair, Completely bounded multilinear maps and $C^{*}$-algebraic cohomology, Invent. Math., 90 (1987), 279-296.

[4] E. Christensen and A. M. Sinclair, Representations of completely bounded multilinear operators, J. Funct. Anal., 72 (1987), 151-181.

[5] E. G. Effros and U. Haagerup, Lifting problems and local reflexivity for $C^{*}$ algebras, Duke Math. J., 52 (1985), 103-128.

[6] E. G. Effros and A. Kishimoto, Module maps and Hochschild-Johnson cohomology, Indiana J. Math., 36 (1987), 257-276.

[7] E. G. Effros and J.-Z. Ruan, Representations of operator bimodules and their applications, J. Operator Theory, 19 (1988), 137-157.

[8] - Multivariable multipliers for groups and their operators algebras, to appear.

[9] P. Eymard, L'algèbre de Fourier d'un groupe localement compact, Bull. Soc. Math. France, 92 (1964), 181-236.

[10] U. Haagerup, Decomposition of completely bounded maps on operator algebras, unpubl. ms. 1980.

[11] - The Grothendieck inequality for bilinear forms on $C^{*}$-algebras, Adv. in Math., 56 (1984), 93-116.

[12] V. I. Paulsen, Completely bounded maps on $C^{*}$-algebras and invariant operator ranges, Proc. Amer. Math. Soc., 86 (1982), 91-96.

[13] V. I. Paulsen and R. R. Smith, Multilinear maps and tensor norms on operator systems, J. Funct. Anal., 73 (1987), 258-276.

[14] Z.-J. Ruan, Subspaces of $C^{*}$-algebras, J. Funct. Anal., 76 (1988), 217-230.

[15] R. R. Smith, Completely bounded maps between $C^{*}$-algebras, J. London Math. Soc. (2), 27 (1983), 157-166.

[16] W. F. Stinspring, Positive functions on $C^{*}$-algebras, Proc. Amer. Math. Soc., 6 (1955), 211-216.

[17] M. Takesaki, Theory of Operator Algebras I, Springer-Verlag, Berlin, 1979.

[18] G. Wittstock, Ein operatorwertiger Hahn-Banach Satz, J. Funct. Anal., 40 (1981), 127-150.

Received June 28, 1988 and in revised form May 1, 1989. This research was partially supported by a grant from the National Science Foundation.

UNIVERSITY OF ILLINOIS

URBANA, IL 61801 



\section{PACIFIC JOURNAL OF MATHEMATICS EDITORS}

\author{
V. S. VARADARAJAN \\ (Managing Editor) \\ University of California \\ Los Angeles, CA 90024-1555-05 \\ Herbert Clemens \\ University of Utah \\ Salt Lake City, UT 84112 \\ Thomas ENRIGHT \\ University of California, San Diego \\ La Jolla, CA 92093
}

R. FINN

Stanford University

Stanford, CA 94305

Hermann FlaschKa

University of Arizona

Tucson, AZ 85721

VAughan F. R. Jones

University of California

Berkeley, CA 94720

Steven Kerckhoff

Stanford University

Stanford, CA 94305
RobION KIRBY

University of California

Berkeley, CA 94720

C. C. MOore

University of California

Berkeley, CA 94720

HAROLd STARK

University of California, San Diego La Jolla, CA 92093

\begin{tabular}{|c|c|c|c|c|}
\hline & ASSOCIA & E EDI & & \\
\hline R. ARENS & $\begin{array}{l}\text { E. F. BECKENBACH } \\
(1906-1982)\end{array}$ & NeumanN & $\begin{array}{c}\text { F. WoLF } \\
(1904-1989)\end{array}$ & K. Yoshida \\
\hline & SUPPORTINC & INSTI & TIONS & \\
\hline UNIVERSITY & ARIZONA & UNIVERS & OF OREGON & \\
\hline UNIVERSITY & BRITISH COLUMBIA & UNIVER & OF SOUTHEI & CALIFORNIA \\
\hline CALIFORNIA & STITUTE OF TECHNOLOGY & STANFOI & UNIVERSITY & \\
\hline UNIVERSITY & CALIFORNIA & UNIVER & OF HAWAII & \\
\hline MONTANA S & TE UNIVERSITY & UNIVER & OF TOKYO & \\
\hline UNIVERSITY & NEVADA, RENO & UNIVER & OF UTAH & \\
\hline NEW MEXIC & TATE UNIVERSITY & WA & N STATE UN & ERSITY \\
\hline OREGON ST & UNIVERSITY & & OF WASHINC & $\mathrm{ON}$ \\
\hline
\end{tabular}




\section{Pacific Journal of Mathematics}

\section{Vol. 143, No. $1 \quad$ March, 1990}

Walter Bergweiler, On the fix-points of composite functions $\ldots \ldots \ldots \ldots \ldots 1$ Aldo Biancofiore, Maria Lucia Fania and Antonio Lanteri, Polarized surfaces with hyperelliptic sections $\ldots \ldots \ldots \ldots \ldots \ldots \ldots \ldots \ldots$

Ciprian Borcea, Deforming varieties of $k$-planes of projective complete

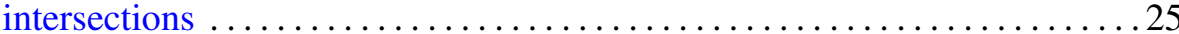

Morton Brown, Fixed points for orientation preserving homeomorphisms of

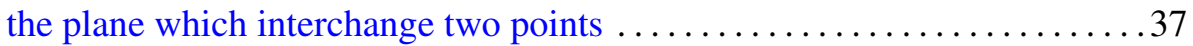

Hao Zhi Chuan, Note on the inequality of the arithmetic and geometric means

Paul Henry Edelman and Dennis E. White, Codes, transforms and the

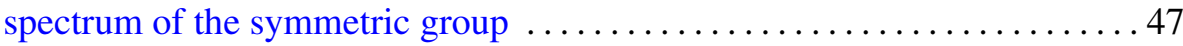

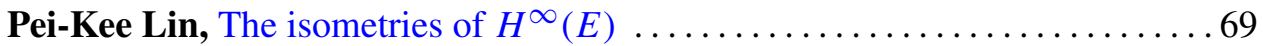

James J. Moloney, Residue class domains of the ring of convergent

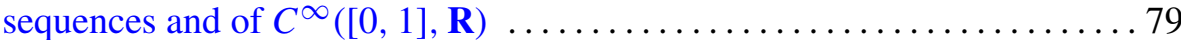

Zhong-Jin Ruan, The structure of pure completely bounded and completely

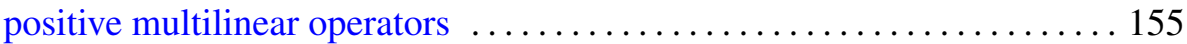

Wolfgang Ruess and William H. Summers, Weakly almost periodic semigroups of operators

Gideon Schwarz, A pretender to the title "canonical Moebius strip" . . . . . . 195

Ryszard Szwarc, Banach algebras associated with spherical representations of the free group 